ARTÍCULO ORIGINAL

Rev Colombiana Cienc Anim 2016; 8(Supl):377-385.

\title{
Frecuencia de patologías oculares en caballos de vaquería en explotaciones ganaderas del departamento de Córdoba, Colombia.
}

\author{
Frequency of ocular pathology in dairy horses in farms in the department of \\ Córdoba, Colombia
}

\author{
MONTES V, DONICER ${ }^{1}$ Ph.D, BUITRAGO M, JHONNY² M.Sc, CARDONAA, JOSÉ2* Ph.D \\ ${ }^{1}$ Universidad de Sucre, Facultad de Ciencias Agropecuarias, Departamento de Zootecnia, \\ Grupo de Investigación en Mejoramiento y Reproducción Animal, Sincelejo, Colombia. \\ ${ }^{2}$ Universidad de Córdoba, Facultad de Medicina Veterinaria y Zootecnia, Grupo de \\ Investigación en Medicina de Grandes Animales (MEGA), Montería, Colombia.
}

\section{Key words:}

Eyes;

blepharitis;

habronemosis:

sarcoid;

ophthalmology;

equine.

(Source: MeSH).

\section{Abstract}

The aim of determine the frequency of occurrence of ocular and periocular diseases that occur in horses dairy department of Cordoba, Colombia. 119 clinical cases of dairy horses of both sexes aged between 3 and 16 years old with ocular and periocular lesions characterized macroscopically were selected. Was found, greater impairment in ocular structures especially corneal pathologies, followed by periocular alterations. The ocular abnormalities most frequently diagnosed in descending order were: not ulcerative keratitis (21\%), ulcerative keratitis (19.3\%), parasitic conjunctivitis Habronema $\operatorname{spp}(16 \%)$, infectious conjunctivitis (12.6\%), anterior uveitis (10.1\%), blepharitis $(8.4 \%)$ chronic superficial keratitis (pannus) $(6.7 \%)$, fibroblastic sarcoid periocular (1.7\%), ocular melanoma, anterior synechiae, cyst corpora nigra, and hipopión panoftalmia $(0.8 \%$ each). The most affected age group was 5 to 10 years (46.2\%), followed by under 5 years old $(28.6 \%)$ and those over 10 years $(25.2 \%)$ respectively, and the most affected sex corresponded to entire males (59.6\%). This is the first report of ophthalmic disorders in horses done in the Department of Cordoba, Colombia and evidence a high presence of ophthalmic disorders in horses dairy, making this work an important contribution on the epidemiological status of various eye disorders in horses work.

\section{Resumen}

El objetivo fue determinar la frecuencia de presencia de las enfermedades oculares y peri oculares que ocurren en los caballos de vaquería del departamento de Córdoba, Colombia. Se tomaron 119 casos clínicos de caballos de vaquería de ambos sexos con edades comprendidas entre 3 y 16 años que presentaron lesiones oculares y peri oculares caracterizadas macroscópicamente. Se encontró una mayor afección en las estructuras oculares en especial las patologías corneales, seguida de las alteraciones peri oculares. Las alteraciones oculares más frecuentemente diagnosticadas en orden descendente fueron: queratitis no ulcerativa (21\%), queratitis ulcerativa $(19,3 \%)$, conjuntivitis parasitaria por Habronema spp (16\%), conjuntivitis infecciosa (12,6\%), uveítis anterior $(10,1 \%)$, blefaritis $(8,4 \%)$ queratitis superficial crónica (pannus) $(6,7 \%)$, sarcoide fibroblástico periocular $(1,7 \%)$, melanoma ocular, sinequia anterior, quiste de la corpora nigra, hipopión y panoftalmia (con $0,8 \%$ cada uno). El grupo etario más afectado fue el de 5 a 10 años (46,2\%), seguido de los menores de 5 años $(28,6 \%$ y los mayores de 10 años $(25,2 \%)$ respectivamente y el sexo más afectado correspondió a los machos enteros (59,6\%). Este es el primer reporte de alteraciones oftálmicas en equinos realizado en el Departamento de Córdoba y Colombia y evidencia una alta presencia de alteraciones oftálmicas en caballos de vaquería, haciendo de este trabajo un importante aporte sobre el estado epidemiológico de las diferentes alteraciones oculares en caballos de trabajo. 


\section{Introducción}

Las enfermedades oculares son comunes en el caballo, estas pueden ser primarias o pueden desarrollarse de manera secundaria a muchas enfermedades sistémicas, frecuentemente estas alteraciones no son evidentes a menos que se realice un examen oftalmológico especifico, por lo que aún existen muchos retos en la oftalmología equina (HUGHES, 2010; BAUER, 2015).

En equinos las enfermedades oftálmicas son diversas en sus etiologías y patogenias comprometiendo la calidad de la visión en grados variables, dependiendo si ocasionan afección de todo el sistema óptico o de estructuras oculares de forma individual (CUNHA et al. 2015), por lo que un examen oftálmico sistemático, combinado con una historia detallada y un adecuado examen físico son esenciales para obtener un diagnóstico temprano y acertado de los trastornos oculares (THANGADURAl et al. 2010; BAUER, 2015). Cuanto más rápidamente es diagnosticada cualquier condición oftálmica mejor será su respuesta al tratamiento y su pronóstico en la mayoría de los casos, ya que el ojo es un órgano sensible y el tiempo es esencial cuando se trata de enfermedades oculares (BAUER, 2015). La terapia indicada y el tiempo de recuperación pueden variar también con factores como el agente etiológico, la estructura afectada y la gravedad de la lesión (CUNHA et al. 2015)

El ojo equino puede ser examinado con equipos portátiles simples y muchas condiciones patológicas pueden ser diagnosticadas con el animal en estación, y puede recurrirse al uso de cámaras digitales para obtener imágenes del segmento anterior y peri orbital para realizar una evaluación más detallada, así como para realizar seguimiento del paciente. Se requiere de experiencia y estudio para discernir la diferencia entre un ojo normal, uno que tiene una variante clínicamente insignificante y el ojo que tiene un problema serio (DWYER, 2012). El reconocimiento de las manifestaciones oculares de enfermedades sistémicas puede permitir dirigir el tratamiento oftálmico con beneficios potenciales para limitar la patología ocular y perdida de la visión (HUGHES, 2010). En los casos en los cuales la enfermedad ocular resulta en ceguera o en un ojo doloroso se requerirá de enucleación, siendo esencial la remisión del globo ocular para evaluación microscópica en la determinación de la causa de la ceguera, permitiendo así tener un pronóstico para el ojo contralateral (BAUER, 2015)

El estudio de las alteraciones oftalmológicas de los equinos se ha centrado en alteraciones particulares como la uveítis recurrente equina, las neoplasias oculares y las ulceras corneales (BROOKS, 2012; BROOKS, 2014; CARVALHO et al. 2014; MONTGOMERY, 2014; WITKOWSKI et al. 2016) y los estudios de prevalencia se han realizado principalmente mediante el análisis de la casuística clínica de hospitales (HURN \& TRUNER, 2006; THANGADURAI et al. 2010; TAMILMAHAN et al. 2013), lo que hace necesario realizar estudios poblacionales ya que estos deben estar más próximos a la realidad, pues el estudio en las poblaciones es independiente de la percepción del problema por parte del propietario y de su disponibilidad de buscar atención (REICHMAN et al. 2008).

Este estudio busca reportar la frecuencia de presentación de diversas alteraciones oftálmicas en caballos de vaquería del departamento de Córdoba; Colombia, diagnosticadas en el Servicio Clínico Ambulatorio de Grandes Animales de la Facultad de Medicina Veterinaria de la Universidad de Córdoba en diferentes explotaciones ganaderas del Departamento, información que podrá ser usada como precedente para futuras investigaciones al ser la primera publicación de este tipo en la región y en el país.

\section{Materiales y Métodos}

El estudio fue realizado en el departamento de Córdoba, ubicado entre las coordenadas $7^{\circ} 23^{\prime}$ y $9^{\circ} 26^{\prime} \mathrm{LN}$ y los $74^{\circ} 52^{\prime}$ y $76^{\circ} 32^{\prime} \mathrm{LO}$, a una altura de $30 \mathrm{msnm}$, con temperatura promedio anual de 28 ${ }^{\circ} \mathrm{C}$, humedad relativa del $82 \%$, precipitación media anual de $1400 \mathrm{~mm}$ y pertenece a la formación climática de bosque tropical lluvioso. Se presentan dos estaciones bien definidas (época de lluvia y época seca) (PABON et al. 2001).

Se realizó un estudio retrospectivo, siendo seleccionados 119 casos clínicos de caballos de vaquería con lesiones oculares macroscópicas diagnosticadas por el Servicio Clínico Ambulatorio de Grandes Animales de la Facultad de Medicina 
Veterinaria de la Universidad de Córdoba entre los meses de septiembre de 2015 y octubre de 2016. Se incluyeron pacientes de ambos sexos, con edades que oscilaron entre 3 y 16 años. Se realizó estadística descriptiva y se clasificaron las patologías encontradas en función del sexo y la edad.

El procedimiento de diagnóstico oftalmológico utilizado por el Servicio Clínico Ambulatorio de Grandes Animales de la Facultad de Medicina Veterinaria de la Universidad de Córdoba consiste en la elaboración de un examen clínico general, en el cual fueron identificadas alteraciones macroscópicas de estructuras oculares y peri oculares, evaluadas clínicamente mediante inspección y palpación, posteriormente cuando se consideró necesario se realizaron métodos diagnósticos complementarios como evaluación de reflejos (respuesta de amenaza, reflejo palpebral, reflejo pupilar directo, reflejo consensual), tinciones diagnosticas (fluoresceína sódica), test de Schrimer y lavados naso lagrimales, también se evaluó la respuesta a la terapéutica aplicada en las diferentes patologías. Todas las lesiones oculares fueron caracterizadas anatomopatológicamente y fotografiadas en alta definición (Sony DSCHX10V, China) para su posterior análisis. Cuando se presentaron lesiones epiteliales tumoriformes se realizaron biopsias de tejido (CARDONA et al. 2013; CARDONA et al. 2014), las cuales fueron fijadas en formalina al $10 \%$, procesadas hasta su inclusión en parafina, cortadas a $5 \mu \mathrm{m}$ de espesor en un micrótomo (Leica RM2125 RTS $®$, Japón) y coloreadas con Hematoxilina - Eosina (HE) en el Laboratorio de Patología Animal del Departamento de Ciencias Pecuarias de la Universidad de Córdoba, Colombia. La información obtenida a partir de las historias clínicas fue diligenciada y procesada en una base de datos elaborada en Microsoft Office Excel 2016 ${ }^{\circledR}$. Se realizó estadística descriptiva, determinando la frecuencia de presentación de cada uno de los diagnósticos obtenidos para los trastornos oculares y se realizó la discriminación por sexo y edad.

Los animales no fueron sometidos a dolor y/o estrés innecesario, por lo que fueron inmovilizados teniendo en cuenta las normas técnicas en el manejo y sujeción de animales, enmarcado en el cumplimiento de la Declaración Universal de los Derechos de los Animales, referente a los principios éticos internacionales para la investigación biomédica con animales del CIOMS (Council for International Organizations of Medical Sciences) establecida por la UNESCO (United Nations Educational, Scientific and Cultural Organization) y la OMS (Organización Mundial de la Salud) de 1949 y de la Ley 84 de Octubre 27 de 1989 (Estatuto Colombiano de Protección Animal) (MRAD, 2006).

\section{Resultados y discusión}

En la tabla 1 se observa la distribución porcentual de cada uno de los diagnósticos oftalmológicos realizados, Siendo observado una mayor afección en las estructuras oculares que en las peri oculares.

Tabla 1. Discriminación de patologías oculares y su porcentaje en 119 caballos de vaquería del Departamento de Córdoba.

\begin{tabular}{cc}
\hline Patologías Oculares & $\mathbf{n}^{\circ}(\%)$ \\
\hline Queratitis no ulcerativa & $25(21,0)$ \\
Queratitis ulcerativa & $23(19,3)$ \\
Habronemosis conjuntival & $19(16,0)$ \\
Conjuntivitis infecciosa & $15(12,6)$ \\
Uveítis anterior & $12(10,1)$ \\
Blefaritis & $10(8,4)$ \\
Queratitis superficial crónica (Pannus) & $8(6,7)$ \\
Sarcoide Fibroblástico periocular & $2(1,7)$ \\
Melanoma ocular & $1(0,8)$ \\
Sinequia anterior & $1(0,8)$ \\
Quiste de la corpora nigra & $1(0,8)$ \\
Hipopión & $1(0,8)$ \\
Panoftalmia & $1(0,8)$ \\
\hline Total & $119(100)$ \\
\hline
\end{tabular}

Entre las patologías corneales, la de mayor frecuencia fue la queratitis no ulcerativa (queratitis superficial) con $21 \%$ (Figura 1c), seguida de ulceras corneales con 19,3\% (Figura 1d), conjuntivitis parasitaria por Habronema spp con $16 \%$, conjuntivitis infecciosa con $12,6 \%$, uveítis anterior con $10,1 \%$, blefaritis con $8,4 \%$ (Figura 2a), queratitis superficial crónica (pannus) con $6,7 \%$ (Figura $2 \mathrm{~b}$ ), sarcoide fibroblástico periocular con $1,7 \%$. Las alteraciones como melanoma ocular, sinequia anterior (Figura 2c), quiste de la corpora 


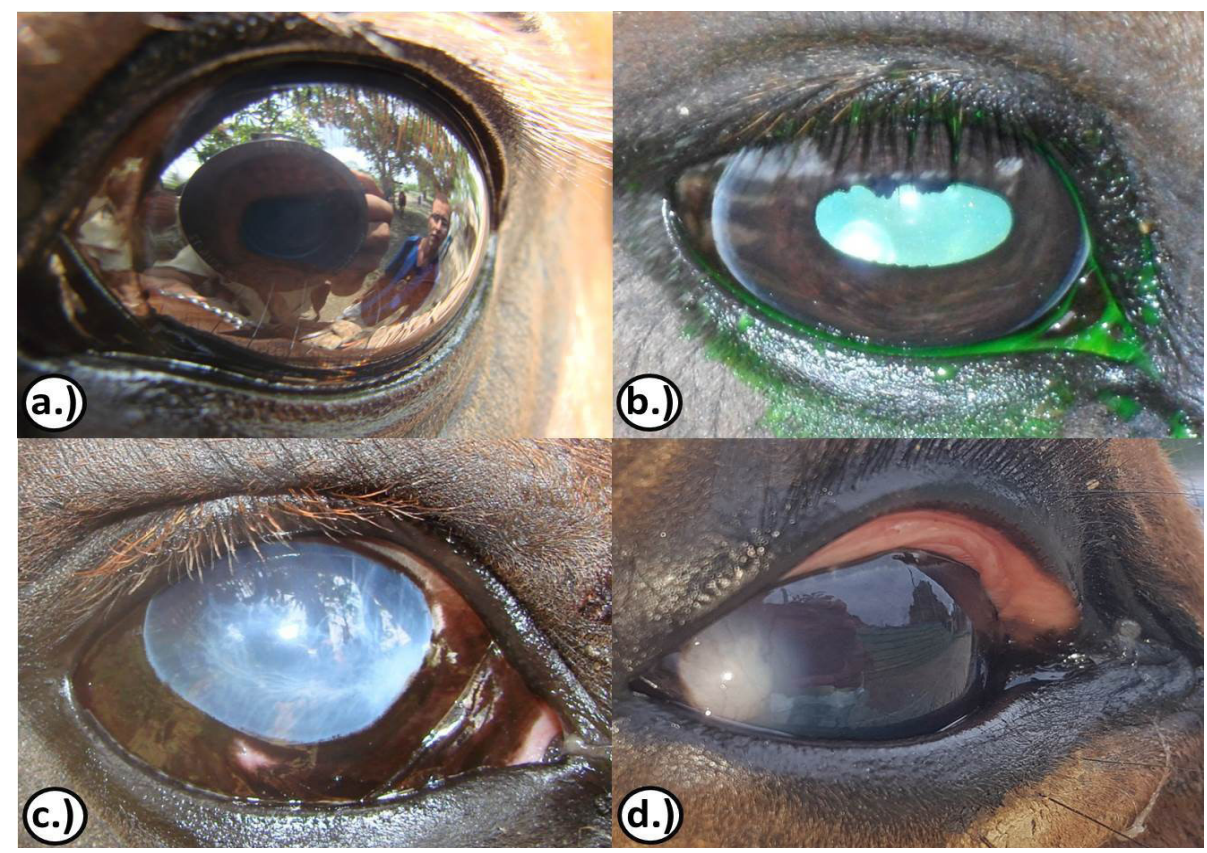

Figura 1. a.) y b.) Ojo con apariencia normal y prueba de la Fluoresceína sódica negativa: Observe el reflejo de objetos y brillo de la córnea y note que no quedan rastros de fluoresceína en la córnea luego de ser lavada. c.) Queratitis superficial: Observe la opacidad difusa de la córnea hasta el límite con la esclerótica. d.) Úlcera corneal: Observe la opacidad del cristalino focalizada de la córnea en su polo posterior

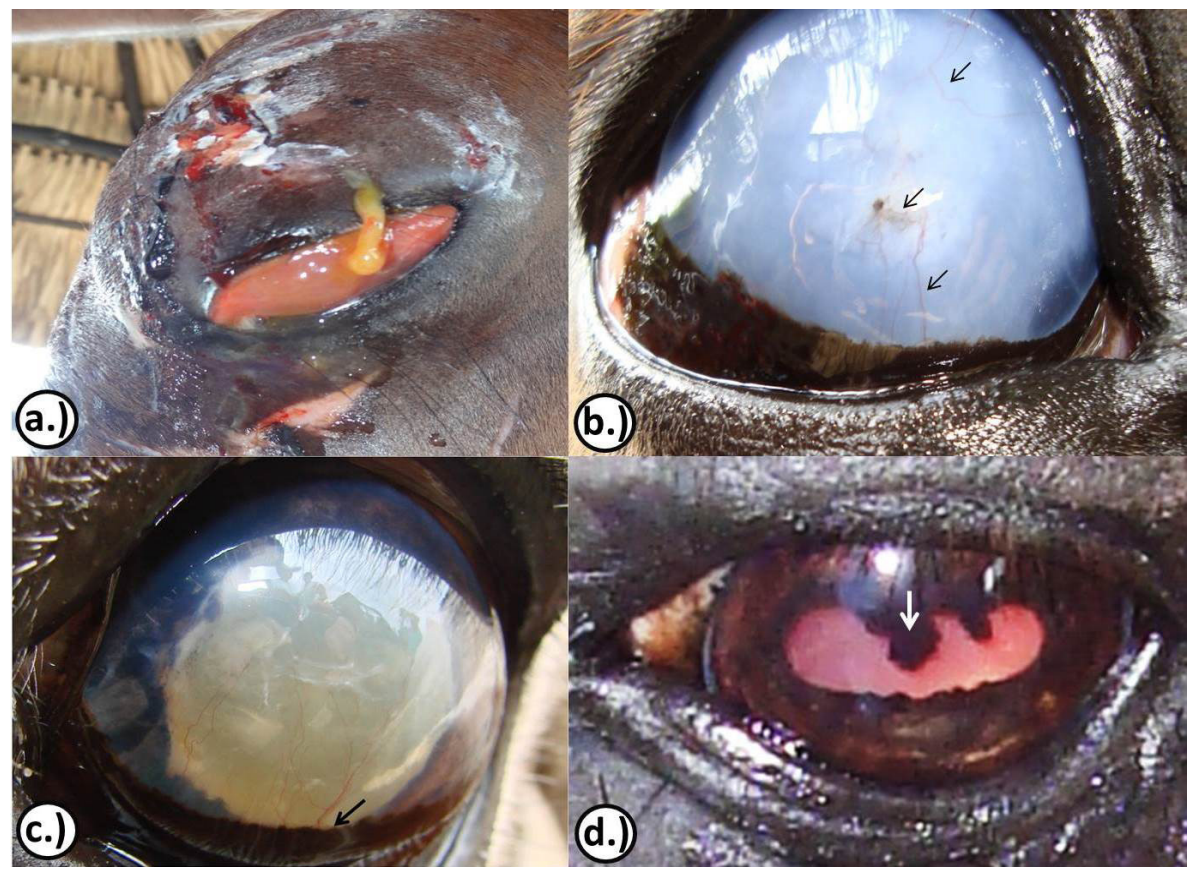

Figura 2. a.) Blefaro conjuntivitis traumática: Observe el aumento de volumen de los párpados con presencia de lesiones y salida de secreción fibrino purulenta. b.) Queratitis pannosa: Observe la opacidad difusa de la córnea con presencia de vasos sanguineos o pannus (flechas). c.) Sinéquia anterior: Observe la opacidad del cristalino y la unión de este al iris en su parte ventral (flecha). d.) Quiste de la corpora nigra: Observe el aumento de volumen de la granula iridis hacia la luz de la pupila (flecha). 
nigra (Figura 2d), hipopión y panoftalmia, se presentan con una frecuencia inferior al $1 \%$ en la población en estudio.

En la tabla 2 se observa la distribución de las patologías oculares por sexo. Se puede observar una mayor frecuencia de presentación de alteraciones oculares en machos $(87,4 \%)$ respecto a las hembras $(12,6 \%)$, siendo afectados principalmente los machos enteros (59,6\%).

Tabla 2. Discriminación de patologías oculares y su porcentaje en 119 Caballos de vaquería del Departamento de Córdoba.

\begin{tabular}{ccccc}
\hline Patologías Oculares & n (\%) & \multicolumn{3}{c}{ Sexo } \\
\cline { 3 - 5 } & & M E & MC & H \\
\hline Queratitis no ulcerativa & $25(21,0)$ & 11 & 10 & 4 \\
Queratitis corneales & $23(19,3)$ & 13 & 9 & 1 \\
Habronemosis conjuntival & $19(16,0)$ & 9 & 6 & 4 \\
Conjuntivitis infecciosa & $15(12,6)$ & 11 & 4 & - \\
Uveítis anterior & $12(10,1)$ & 8 & 2 & 2 \\
Blefaritis & $10(8,4)$ & 10 & - & - \\
Queratitis superficial crónica (Pannus) & $8(6,7)$ & 6 & 2 & - \\
Sarcoide Fibroblástico periocular & $2(1,7)$ & 2 & - & - \\
Melanoma ocular & $1(0,8)$ & - & - & 1 \\
Sinequia anterior & $1(0,8)$ & 1 & - & - \\
Quiste de la corpora nigra & $1(0,8)$ & - & - & 1 \\
Hipopión & $1(0,8)$ & - & - & 1 \\
Panoftalmia & $1(0,8)$ & - & - & 1 \\
\hline Total & $\mathbf{1 1 9}$ & $\mathbf{7 1}$ & $\mathbf{3 3}$ & $\mathbf{1 5}$ \\
\hline
\end{tabular}

ME(Machos enterros), MC (Machos castrados), H(hembras).

En la tabla 3 se muestra la distribución de las patologías oculares por edad. La mayor frecuencia se encontró en los animales entre 5 a 10 años de edad $(46,2 \%)$, seguida de los animales mayores de 10 años $(25,32 \%)$.

Estudios realizados a nivel mundial muestran que el equino se encuentra dentro de las especies animales más susceptibles a padecer de alteraciones oculares (TAMILMAHAN et al. 2013; MARTINS \& BARROS, 2014). En Australia, en caballos de carreras encontraron que solo 32,4 $\%$ de los animales evaluados presentaba un examen oftalmológico completamente normal, y un $67,6 \%$ de los animales presentaba alteraciones oftalmológicas (HURN \& TRUNER, 2006).
Tabla 3. Discriminación de patologías oculares y su porcentaje en 137 animales con algún tipo de patología.

\begin{tabular}{|c|c|c|c|c|}
\hline \multirow{2}{*}{ Patologías oculares } & \multirow{2}{*}{$\mathbf{n}^{\circ}(\%)$} & \multicolumn{3}{|c|}{ EDAD (Años) } \\
\hline & & $<5$ & 5 a 10 & $>10$ \\
\hline Queratitis no ulcerativa & $25(21,0)$ & 6 & 12 & 7 \\
\hline Queratitis ulcerativa & $23(19,3)$ & 3 & 15 & 5 \\
\hline Habronemosis conjuntival & $19(16,0)$ & 2 & 11 & 6 \\
\hline Conjuntivitis infecciosa & $15(12,6)$ & 5 & 4 & 6 \\
\hline Uveítis anterior & $12(10,1)$ & 9 & 1 & 2 \\
\hline Blefaritis & $10(8,4)$ & 7 & 2 & 1 \\
\hline $\begin{array}{l}\text { Queratitis superficial crónica } \\
\text { (Pannus) }\end{array}$ & $8(6,7)$ & 1 & 6 & 1 \\
\hline Sarcoide Fibroblástico periocular & $2(1,7)$ & - & 2 & - \\
\hline Melanoma ocular & $1(0,8)$ & - & - & 1 \\
\hline Sinequia anterior & $1(0,8)$ & - & 1 & - \\
\hline Quiste de la corpora nigra & $1(0,8)$ & - & 1 & - \\
\hline Hipopión & $1(0,8)$ & 1 & - & - \\
\hline Panoftalmia & $1(0,8)$ & - & - & 1 \\
\hline Total & $\begin{array}{c}119 \\
(100 \%)\end{array}$ & $\begin{array}{c}34 \\
(28,6 \%)\end{array}$ & $\begin{array}{c}55 \\
(46,2 \%)\end{array}$ & $\begin{array}{c}30 \\
(25,2 \%)\end{array}$ \\
\hline
\end{tabular}

Aunque las enfermedades oculares son comunes en el equino, solo el 3,3\% de los propietarios reportan problemas oculares en sus caballos, siendo la descarga ocular el motivo de consulta mas comun, y solo $2,6 \%$ perciben la vision como un importante tema de salud, por lo que existe un gran numero de caballos que pueden presentar problemas oculares sin diagnosticar, lo que pueden ser una fuente de un desconfort croico de bajo grado para el animal (BAUER, 2015; MALALANA, 2016) y que si no son tratadas a tiempo y de forma adecuada las alteraciones oculares pueden terminar en ceguera, por lo que la principal importancia de las enfermedades oftálmicas es el grado de compromiso visual que puede inutilizar a los animales para cumplir con el trabajo destinado (REICHMAN et al. 2008), por lo que puede tener efectos nocivos (deletéreos) tanto en los caballos como en sus jinetes, siendo necesario incluir el examen ocular como una parte del examen físico de rutina en la práctica equina (THANGADURAl et al. 2010).

En el presente estudio se encontró una mayor afección de las estructuras oculares que de las peri 
oculares, es así como en estudios retrospectivos realizados en Brasil a nivel de laboratorio histopatológico encontraron que el $1,6 \%$ de los exámenes histopatológicos de diversas especies domesticas correspondían a lesiones oculares y peri oculares, reportando una mayor prevalencia a nivel de parpados, seguidos del tercer parpado y la conjuntiva, para el caso de los caballos la alteración más frecuente se debió a neoplasias siendo principalmente sarcoide, seguido del tumor de células escamosas (MARTINS \& BARROS, 2014). Estos resultados pueden darse debido a que pocas alteraciones oftalmológicas en el equino requieren de diagnóstico histopatológico, siendo este método usado para las alteraciones de tipo neoplásico, que usualmente presentan una ubicación periocular (MONTGOMERY, 2014), o en los casos de enucleación ocular en los que se remite el ojo completo para realizar el diagnóstico definitivo (BAUER, 2015).

Los tumores oculares en equinos pueden ser categorizados basados en su localización anatómica, cada localización anatómica posee un diferencial de tumores único, factores pronósticos y consideraciones terapéuticas dependientes del tipo de tumor (MONTGOMERY, 2014)

Al analizar la distribución de los diagnósticos realizados se observo un predominio de la queratitis no ulcerativa (queratitis superficial) con un $21 \%$, seguido de las queratitis ulcerativas con un $19,3 \%$, la conjuntivitis parasitaria por Habronema spp con un $16 \%$, conjuntivitis infecciosa en un $12,6 \%$ y la uveítis anterior con el $10 \%$. Estos hallazgos difieren de los hallados en otros lugares del mundo (India), , en donde estudios retrospectivos a nivel de clínica reportaron que la opacidad corneal era la alteración con mayor frecuencia de presentación, seguida de Setariasis ocular y lesiones traumáticas (TAMILMAHAN et al. 2013), mientras que en Australia las alteraciones de la retina $(57,4 \%)$ y el cristalino $(19,6 \%)$ fueron las más observadas (HURN \& TRUNER , 2006). A nivel de Suramérica estudios retrospectivos realizados en Brasil encontraron en équidos de tracción el $48 \%$ de las alteraciones oculares se debían a alteraciones corneales (cataratas $24 \%$ ), alteraciones conjuntivales $(24 \%)$ y uveítis recurrente $(8 \%)$, siendo el trauma el principal causante de las enfermedades oftalmológicas (REICHMAN et al. 2008), mientras que en este mismo país un estudio realizado en un centro de referencia clínica entre los años 2011 y 2014 determino que de los casos que se presentaron a consulta oftalmológica el $45,7 \%$ correspondían a ulceras corneales, $14,2 \%$ a abscesos estromales, $8,5 \%$ a uveítis recurrente, $20 \%$ perforación de globo ocular y el $11,4 \%$ presentaban carcinoma de células escamosas (CUNHAet al. 2015). En potros neonatos remitidos a hospitales por condiciones no oculares se encontró que la mayoría $(55,7 \%)$ de pacientes con infección sistémica tenían al menos una lesión oftálmica. Dentro de las anormalidades encontradas se incluyen conjuntivitis $(42,9 \%)$ uveítis $(25,7 \%)$, queratitis ulcerativa $(18,6 \%)$ queratitis no ulcerativa $(143 \%)$ entropión $11,4 \%)$ y cataratas $(8,6 \%)$ (Labelle, 2011).

Esto muestra que existe una diversidad de etiologías que varían en función de la localización geográfica de los animales y su función zootécnica, pues expone a los pacientes a distintos factores de riesgo, sin embargo deben realizarse estudios posteriores, ya que los estudios de prevalencia se han realizado principalmente mediante el análisis de la casuística clínica de hospitales y de manera retrospectiva, lo que hace que en parte la casuística dependa del propietario y su percepción del problema, por lo que deben realizarse estudios prospectivos poblacionales que se ajustan más a la realidad del problema ya que son independientes de la percepción del problema por parte del propietario y de su disponibilidad de buscar atención (REICHMAN et al. 2008)

De forma similar a lo encontrado en este estudio TAMILMAHAN et al. (2013) encontro una mayor predisposicion a las patologias oculares en machos, esto podria deberse a que los machos, especialmente los sementales pueden ser mas activos y de mas dificil manejo lo que puede favorecer la presentacion de lesiones de tipo traumatico, mientras que las hembras, especialmente aquellas en estado gravidico son mas calmadas y tratadas con mayor cuidado, sin embargo se recomienda que se realicen estudios posteriores para dilucidar mas claramente el efecto del sexo en la presentacion de patologias oculares.

Respecto a la edad, TAMILMAHAN et al. (2013) encontraron que los animales más afectados se encontraban en el rango de 1 a 5 años, mientras que MALALANA (2016), indica que las anormalidad oculares son un hallazgo comun en caballos geriatras y que algunas de estas anomalías son específicas de 
caballos viejos, mientras que otras son el resultado de trastornos previos o inflamaciones que iniciaron más temprano en la vida pero que se vuelven más evidentes cuando el daño ha avanzado. En este estudio se obtuvo que los animales más afectados se encontraban entre los 5 y 10 años, esto puede ser efecto de la función zootécnica, pues al ser animales de vaquería la mayor parte de la población activa se encuentra en este rango de edad, siendo bajo el uso de animales menores de cinco años y mayores de diez debido a la falta de adiestramiento o a la disminución de su resistencia física.

La cornea fue la estructura ocular que agrupo la mayor cantidad de diagnósticos en este estudio con un $47 \%$ de las patologías encontradas, las queratopatias pueden ser ulcerativas o no ulcerativas, infecciosas o estériles. Las queratopatias infecciosas en los caballos pueden ser causadas por bacterias, hongos y posiblemente virus, las estériles pueden ser causadas por cuerpos extraños, problemas de la película lagrimal, denervación corneal o distrofias de la membrana basal corneal (BROOKS et al. 2014).

Clínicamente las queratiti ulcerativa es la las enfermedad oftálmicas más comúnes (singularplural) del caballo.Al ser una enfermedad amenazante de la visión requiere un diagnóstico clínico temprano y una apropiada terapia médica. (BROOKS, 2012; BROOKS, 2014; KIRK, 2014). La queratitis ulcerativa puede presentarse inicialmente como una ulcera epitelial menor o infiltrados celulares con dolor leve, blefaroespasmo epifora y fotofobia, la leve perdida de pestañas del parpado superior puede ser un signo temprano y sutil de ulceración corneal. Al inicio la uveítis anterior y la vascularización corneal pueden no ser clínicamente pronunciadas. El diagnostico se realiza mediante la retención de fluoresceína. (BROOKS, 2014).

Las ulceras corneales pueden ir de simples rupturas superficiales, abrasiones del epitelio corneal, derretimiento del colágeno corneal, hasta perforación corneal de espesor completo con prolapso de iris, siendo posibles secuelas la ruptura del globo ocular, phthisis bulbi y ceguera. El ojo prominente del caballo puede predisponer a ulcera corneal de tipo traumático. (BROOKS, 2012; BROOKS, 2014). En este estudio las principales alteraciones corneales correspondieron a la queratitis no ulcerativa, queratitis ulcerativa y la queratitis superficial crónica (Pannus).
Un $28 \%$ de los diagnósticos correspondieron a alteraciones de la conjuntiva, se ha reportado que la conjuntivitis es un hallazgo inespecífico que indica inflamación ocular pero también puede ser vista en enfermedades sistémicas o secundarias a enfermedades de la córnea, esclera, úvea anterior, sistema naso lagrimal y órbita, (BROOKS, 2014), por lo que es un diagnóstico común pero debe siempre acompañarse de investigaciones más profundas para determinar la causa inicial de la reacción inflamatoria. Las enfermedades primarias de la conjuntiva incluyen cuerpos extraños, parásitos, ojo seco, enfermedades virales neoplasia, trauma o condiciones alérgicas; Para diferenciar la conjuntivitis primaria de otras causas secundarias más severas es esencial realizar un examen oftalmológico completo para determinar las alteraciones oculares que puedan estar causando la enfermedad conjuntival (CARASTRO, 2004; BAUER, 2015) () En este estudio los principales diagnósticos asociados a la conjuntiva fueron la conjuntivitis infecciosa y la conjuntivitis parasitaria por Habronema spp.

Las conjuntivitis parasitarias incluyen dentro de sus principales agentes etiológicos Habronema spp y Thelazia lacrymalis. La habronemosis es una enfermedad parasítica que en su forma conjuntival puede causar granulomas oculares y peri oculares, mientras que Thelazia lacrymalis se ha reportado como un parasito comensal del fornix conjuntival y los ductos naso lagrimales (BROOKS, 2014)

\section{Conclusión}

Este es el primer reporte de alteraciones oftálmicas en equinos realizado en Colombia. Las alteraciones del sistema oftálmico son comunes en los caballos de vaquería, existe una predisposición a este tipo de lesiones en machos enteros y animales entre los 5 y 10 años de edad. Son necesarios estudios posteriores a nivel poblacional para conocer la prevalencia de las alteraciones oftálmicas en equinos del departamento de Córdoba, así como sus factores de riesgo para poder realizar planes de prevención $y$ control. 


\section{Referencias}

BAUER, B. 2015. Ocular pathology. Vet Clin Equine, 31, 425-448.

BROOKS, D. 2012. Equine Corneal Ulceration. Proceedings of the AAEP Focus on Opthalmology, (págs. 1-11). Raleigh, NC, USA.

BROOKS, D. 2014. How to Reach the Medical Standards of Care for Ulcerative and Non-Ulcerative Equine Keratopathies. AAEP PROCEEDINGS, 60, 11-15.

BROOKS, D. 2014. How to Use the Clinical Examination to Determine the Significance of Abnormalities of the Horse Cornea and Adnexa. AAEP PROCEEDINGS, 60, 19-24.

CARASTRO, S. 2004. Equine ocular anatmy and ophtalmic examnation. vet clin equine, 20, 285-299.

CARDONA, J; VARGAS, M. PERDOMO, S. 2013. Estudio clínico e histopatológico del sarcoide fibroblastico en burros (Equus asinus) en Colombia. . Revista Científica FCV-LUZ, XXIII(2), 97-104.

CARDONA, J; VARGAS, M. PERDOMO, S. 2014. Frecuencia de pythiosis cutánea en caballos de producción en explotaciones ganaderas de Córdoba, Colombia. Rev. Med. Vet. Zoot, 61(1), 31-43.

CARVALHO, F; DANTAS, A; RIET-CORREA, F; ANDRADE, R; NETO, P; NETO, E. 2014. Estudo retrospectivo das neoplasias em ruminantes e equídeos no semiárido do Nordeste Brasileiro. Pesq. Vet. Bras., 34(3), 211-216.

CUNHA, F; DA ROSA, B; SOARES, L; PAZINATO, F; SOARES, P; WYNE, C. 2015. Alterações do sistema oftálmico em equinos com ênfase em medidas terapêuticas. Acta Scientiae Veterinariae, 43(suplemento 1), 99-106.

DWYER, A. 2012. Ophthalmology in Equine Ambulatory Practice. Vet Clin Equine, 28, 155-174.

HUGHES, K. 2010. Ocular manifestations of systemic disease in horses. EQUINE VETERINARY JOURNAL, 37, 89-96.

HURN, S; TRUNER, A. 2006. Ophthalmic examination findings of thoroughbred racehorses in australia. veterinary ophthamology, 9(2), 95-100.

KIRK, N. 2014. Essentials of Veterinary Ophthalmology (tercera edicion ed.). Gainesville, Florida, USA: Wiley blackwell.

MALALANA, F. 2016. Ophthalmologic Disorders in Aged Horses. Vet Clin Equine, 32, 249-261.

MARTINS, T; BARROS, C. 2014. Fifty years in the blink of an eye: a retrospective study of ocular and periocular lesions in domestic animals. Pesq. Vet. Bras, 34(12), 1215-1222.

MRAD, A. 2006. Etica en la investigacion con modelos animales experimentales. alternativas y las $3 R$ de Russel una responsabilidad u un compromiso etico que nos compete a todos. Revista colombiana de boetica, 1, 163-184.

MONTGOMERY, K. 2014. Equine ocular neoplasia: A review. Equine Veterinary Education, 26(7), 372-380. Labelle, A. 2011. Ophthalmic lesions in neonatal foals evaluated for nonophthalmic disease at referral hospitals. JAVMA, 239(4), 486-492. 
PABON, J; ESLAVA, J; GOMEZ, R. 2001. Generalidades de la distribucion espacial y temporal de la temperatura del aire y de la precipitacion en Colombia. Meteorologia colombiana, 4, 47-59.

REICHMAN, P; DE OLIVEIRA, A; CARONATO, T. 2008. Ocurrence of ophthamologic diseases in horses used for urban cart hauling in londrina, PR, Brazil. Ciencia Rural Santa Maria, 38(9), 2525-2528.

TAMILMAHAN, P; ZAMA, M; PATHAK, R; MUNEESWARAN, N; KARTHIK, K. 2013. A retrospective study of ocular occurrence in domestic animals: 799 cases. Veterinary World, 6(5), 274-276.

THANGADURAI, R; SHARMA, S; BALI, D; MAHAJAN, V; SAMANTA, I; HAZRA, S. 2010. Prevalence of ocular disorders in an indian population of horses. journal of equine veterinary science, 30(6), 326-329.

WITKOWSKI, L; CYWANSKA, A; PASCHALIS-TRELA, K; CRISMAN, M; KITA, J. 2016. Multiple etiologies of equine recurrent uveitis - A natural model for human autoinmune uveitis:a brief review. comparative inmunology, microbiology and infectious diseases, 44, 14-20. 